\title{
Mass transport due to oscillatory flow through a prestressed viscoelastic tube with a retentive and absorptive wall
}

\author{
Ye Ma, Chiu-On $\mathrm{Ng}^{1}$ \\ Department of Mechanical Engineering, \\ The University of Hong Kong, Pokfulam Road, Hong Kong
}

November 16, 2010

\begin{abstract}
An asymptotic analysis is presented for the mass transport of a solute due to volume-cycled oscillatory flow in a prestressed and viscoelastic tube with a reactive wall layer (wall retention and absorption). Based on the homogenization technique, the convection-diffusion transport equation is derived for the developed time-mean concentration, containing the effective transport coefficients of advection and dispersion as functions of oscillation frequency, initial stresses, viscoelasticity of the wall, and the two wall reactions. The present model as applied to gas transport in pulmonary airways under high-frequency ventilation is examined in detail, through comparison with a model in the literature. The dispersion coefficient is independent of the wall properties if the tube is tethered. The gas transport rate is found to be enhanced monotonically when either the reversible phase partitioning or its exchange rate increases.
\end{abstract}

Keywords: Taylor dispersion; high-frequency ventilation; homogenization technique.

\footnotetext{
${ }^{1}$ Corresponding author. Fax: +852 2858 5415; E-mail: cong@hku.hk.
} 


\section{Introduction}

For patients suffering from acute respiratory failure, e.g., lung injury and acute respiratory distress syndrome, the mechanical ventilator is a common type of device used to assist breathing. Conventional ventilators which mimic normal breathing may lead to lung injury owing to high airway pressures $[1,2]$. High-frequency ventilation (HFV) is a more desirable technique by which a smaller tidal volume of air is pumped into the human lung at a higher frequency by a smaller pressure difference. Gas exchange efficiency is a key factor for the clinical application of HFV, and therefore it is important to study gas transport in pulmonary airways under the operating conditions of $\mathrm{HFV}$.

Gas transport during HFV is largely accomplished by Taylor dispersion [1], instead of convection alone as in conventional ventilators. Taylor dispersion resembles molecular diffusion as a phenomenon, but it arises from an interaction between radial diffusion and axial velocity shear. Depending on the flow, dispersion can be much more efficient than molecular diffusion in spreading a solute in flow through a tube.

The study of longitudinal dispersion of a solute due to a volume-cycled oscillatory flow through a flexible tube is of considerable interest for its many applications in bio-transport processes. Taylor [3] pioneered a fundamental study on dispersion in laminar Poiseuille flow through a rigid straight tube. He pointed out that at a developed state, the dispersion of the slug of a solute along the tube can be treated such that the center of the slug travels with the cross-sectional mean speed of flow (advection velocity), and the slug is diffused with an augmented effective diffusivity (dispersion coefficient) which is a function of the molecular diffusivity and advection velocity. Following Taylor's analysis, Chatwin [4] and Watson [5] analytically solved for the dispersion of a passive contaminant in oscillatory flow driven by a timeperiodic pressure gradient. Here, a pulmonary airway is much more complicated in terms of geometry. Instead of a straight uniform rigid tube, it is in fact tapered $[6,7]$, curved $[8,9,10]$, branched $[11,12]$, and flexible-walled $[13,14,15]$. These geometrical

features each play different roles in controlling the rate of transport of gases in the airways.

The wall flexibility can have significant effects on the gas exchange efficiency, 
where airway flexibility varies among people at different ages or in different lung disease states [1]. The lung compliance may be increased owing to emphysema or aging, but can be reduced for patients with fibrosis or respiratory distress syndrome. Dragon and Grotberg [13], which is hereinafter referred to as DG91, investigated the oscillatory flow and mass transport of gases through a thin-walled viscoelastic tube in the context of HFV. A much more comprehensive flow model was recently developed by $\mathrm{Ma}$ and $\mathrm{Ng}$ [16], who investigated oscillatory as well as time-mean motions of a viscous fluid in a thick-walled flexible tube. The time-mean flow, also called steady streaming, is a second-order steady current when the first-order velocity components are not $\pi / 2$ out of phase. It amounts to a net drift of particles even though the forcing is purely oscillatory. Based on the assumption of small-amplitude waves, Ma and Ng [16] analytically solved for the oscillatory flow and steady streaming as functions of the oscillation frequency (represented by the Womersley number), wall viscoelasticity and initial stresses. The availability of this flow model has made it possible to further study the relation between the wave propagation and gas transport in a flexible tube, which is the objective of the present work.

The mass transport problem studied by DG91 [13] is germane to the one presented in this paper. There are, however, some basic differences that distinguish the present work from DG91 [13], as explained in the appendix.

One specific aim of the present work is to study the developed mass transport of a dissolved species in small-amplitude oscillatory flow through a prestressed, viscoelastic and reactive tube. The tube is assumed to be long, straight, axially and radially stressed, and with a uniform cross-section initially. The outer surface of the tube is assumed to be constrained by the surrounding tissues, and it is hence called a tethered tube. Effects of tapering, curvature, branching and gravity are neglected. The oscillatory and time-mean velocity fields derived by $\mathrm{Ma}$ and $\mathrm{Ng}[16]$ will be followed. A first-order kinetic model is used to describe the reversible reaction due to phase exchange between the fluid and the wall layer, and also the irreversible reaction due to absorption into the wall. By the homogenization technique, the effective transport coefficients of advection and dispersion of the developed timemean concentration in the convection-diffusion transport equation will be derived as functions of oscillation frequency, initial stresses, viscoelasticity of the wall, and the 
rates of the two wall reactions. The early-stage transient concentrations are ignored in this work. The time-scale for the irreversible absorption is assumed to be much longer than that for the reversible phase exchange, which is comparable with the short time-scale of an oscillation period. When discussing the results, we shall first compare with DG91 [13] the mass transport rate in an inert tube. Next, the dependence of the mass transport rate and dispersion coefficient on the wall flexibility will be examined in detail. Finally, the effects of the wall elasticity, initial stresses, wall reactions on the advection velocity and the transport rate are also discussed.

\section{Mathematical formulation}

The mass transport induced by a small-amplitude periodic wave traveling along a long flexible tube of circular cross-section is considered. The core of the tube, which has an initially inflated radius $a$, is filled with an incompressible Newtonian viscous fluid. The wall of the tube, whose outer radius is initially $b(b>a)$, is supposed to be made up of a Voigt type of material. Eulerian cylindrical coordinates are used in which the radial and axial coordinates are denoted by $r$ and $z$, respectively. Axisymmetry is assumed, and hence any dependence on the azimuthal position is eliminated. The outer surface of the tube is assumed to be zero-displacement axially (i.e., a tube constrained by the surrounding tissues). A progressive wave of wavenumber $k$ is induced in the system by an oscillatory pressure gradient of angular frequency $\sigma$ :

$$
p_{f 1}=\operatorname{Re}\left[p_{s} e^{i(\sigma t-k z)}\right]
$$

where $p_{f 1}$ represents the oscillatory fluid pressure whose amplitude is $p_{s}, t$ is time,

and $i=\sqrt{-1}$ is the complex unit. The mathematical expressions for the oscillatory flow and the induced steady streaming, which have been derived by Ma and Ng [16], will be given later.

Having known the fluid motion in the system, we may now consider the transport of a species, which is completely miscible with the fluid in the tube, and may also undergo heterogeneous reactions at the tube wall. The convection-diffusion equation for the transport of a reactive solute with molecular diffusivity $D$ can be written as 
follows:

$$
\frac{\partial C}{\partial t}+u \frac{\partial C}{\partial r}+w \frac{\partial C}{\partial z}=D \frac{\partial^{2} C}{\partial z^{2}}+\frac{D}{r} \frac{\partial}{\partial r}\left(r \frac{\partial C}{\partial r}\right), \quad 0<r<a+\xi,
$$

together with the boundary conditions $[17,18,19]$ :

$$
\begin{gathered}
\frac{\partial C}{\partial r}=0, \quad r=0, \\
-D \nabla C \cdot \vec{n}-\Gamma C=\frac{\partial C_{s}}{\partial t}=\kappa\left(\omega C-C_{s}\right), \quad r=a+\xi,
\end{gathered}
$$

where $u$ and $w$ are the radial and axial fluid velocities, $C(r, z, t)$ is the concentration (mass of species per bulk volume of fluid) of the fluid phase, and $C_{s}=C_{s}(z, t)$ is the concentration (mass of species retained per unit surface area of the wall) of the solid phase. The wall reactions are specified on the moving surface $r=a+\xi$, where $\xi=$ $\xi(z, t)$ is the radial deformation of the fluid-wall interface, and $\vec{n}$ is the unit outward normal to this interface. Here, $\Gamma, \kappa$ and $\omega$ are the irreversible absorption rate, the reversible reaction rate and the partition coefficient, respectively. The first equality in Eq. (4) describes the irreversible reaction by which the substance is transformed and absorbed irreversibly into the wall. The second equality is for the reversible exchange between phases of the species across the fluid-lining interface.

Further assumptions are made to prepare ground for the perturbation analysis. First, we suppose that the length-scale $L$ for the axial spreading of the species is much greater than the tube radius $a$. Therefore, the following small parameter

$$
\epsilon=a / L \ll 1
$$

is used as an ordering parameter. Second, the oscillation period of the flow is so short that the diffusion across the entire section may be accomplished, but there is no appreciable transport effect down the tube within this short time-scale. Third, the two wall reactions are effective over different time scales. The irreversible absorption takes place at a much slower rate than the reversible phase exchange, which can be largely achieved over a finite number of oscillations. Fourth, due to the sharp contrast in length scales defined in Eq. (5), the axial diffusion/dispersion requires an even longer time to be effective. Under these assumptions, three distinct time scales may be introduced [17],

$$
T_{0}=2 \pi / \sigma=O\left(a^{2} / D\right)=O\left(\kappa^{-1}\right)
$$




$$
\begin{aligned}
& T_{1}=O(a \Gamma)=\epsilon^{-1} T_{0}, \\
& T_{2}=L^{2} / D=\epsilon^{-2} T_{0} .
\end{aligned}
$$

Based on these time-scales, we may introduce accordingly

$$
t_{o}=t, \quad t_{1}=\epsilon t, \quad t_{2}=\epsilon^{2} t,
$$

which are, respectively, the fast, medium and slow time variables.

Waves are assumed to be of long wavelength and small amplitude such that $k^{-1}=$ $O(L)$ and the amplitude of the oscillatory pressure $p_{s}$ is one order of magnitude smaller than the static fluid pressure. Based on the shallowness, let us now introduce the following normalized variables, which are distinguished by a caret:

$$
\left.\begin{array}{r}
t=\sigma^{-1} \hat{t}, \quad(r, b)=a(\hat{r}, \hat{b}), \quad \xi=\epsilon a \hat{\xi}, \quad z=L \hat{z}, \\
p_{s}=\epsilon \rho_{f} \sigma^{2} L^{2} p^{o} \quad u=\epsilon L \sigma \hat{u}, \quad w=L \sigma \hat{w}, \quad k=L^{-1} \hat{k}, \\
C=C^{*} \hat{C}, \quad C_{s}=a C^{*} \hat{C}_{s}, \quad \kappa=\sigma \hat{\kappa}, \quad \Gamma=\epsilon a \sigma \hat{\Gamma}, \quad \omega=a \hat{\omega},
\end{array}\right\}
$$

where $\rho_{f}$ is the fluid density, $C^{*}$ is a reference concentration.

According to the normalized variables above, Eqs. (2)-(4) can be expressed as

$$
\begin{gathered}
\operatorname{Pe}\left[\frac{\partial \hat{C}}{\partial \hat{t}}+\hat{u} \frac{\partial \hat{C}}{\partial \hat{r}}+\hat{w} \frac{\partial \hat{C}}{\partial \hat{z}}\right]=\epsilon^{2} \frac{\partial^{2} \hat{C}}{\partial \hat{z}^{2}}+\frac{1}{\hat{r}} \frac{\partial}{\partial \hat{r}}\left(\hat{r} \frac{\partial \hat{C}}{\partial \hat{r}}\right), \quad 0<\hat{r}<1 \\
\frac{\partial \hat{C}}{\partial \hat{r}}=0, \quad \hat{r}=0 \\
-\frac{1}{\mathrm{Pe}}\left(\frac{\partial \hat{C}}{\partial \hat{r}}+\epsilon \hat{\xi} \frac{\partial^{2} \hat{C}}{\partial \hat{r}^{2}}+\epsilon^{2} \frac{\hat{\xi}^{2}}{2} \frac{\partial^{3} \hat{C}}{\partial \hat{r}^{3}}\right)-\epsilon \hat{\Gamma}\left(\hat{C}+\epsilon \hat{\xi} \frac{\partial \hat{C}}{\partial \hat{r}}+\epsilon^{2} \frac{\hat{\xi}^{2}}{2} \frac{\partial^{2} \hat{C}}{\partial \hat{r}^{2}}\right)+O\left(\epsilon^{3}\right) \\
=\frac{\partial \hat{C}_{s}}{\partial \hat{t}}=\hat{\kappa}\left[\hat{\omega} \hat{C}-\hat{C}_{s}+\epsilon \hat{\xi}\left(\hat{\omega} \frac{\partial \hat{C}}{\partial \hat{r}}-\frac{\partial \hat{C}_{s}}{\partial \hat{r}}\right)+\epsilon^{2} \frac{\hat{\xi}^{2}}{2}\left(\hat{\omega} \frac{\partial^{2} \hat{C}}{\partial \hat{r}^{2}}-\frac{\partial^{2} \hat{C}_{s}}{\partial \hat{r}^{2}}\right)\right] \\
\hat{r}=1
\end{gathered}
$$

where $\mathrm{Pe}=\sigma a^{2} / D \leq O(1)$ is the Péclet number. Note that, in Eq. (13), the conditions at the exact position $\hat{r}=1+\epsilon \hat{\xi}$ have been approximated by those at $\hat{r}=1$ using Taylor series expansions. The problem can reduce to the non-reactive or inert case when $\hat{\Gamma}=\hat{\omega}=0$.

Following the asymptotic expansion introduced by Fife and Nicholes [20] and ignoring the transient terms of the early stage, the developed concentrations can be 
expressed by:

$$
\begin{gathered}
\hat{C}(\hat{r}, \hat{z}, \hat{t})=\hat{C}_{0}\left(\hat{r}, \hat{z}, \hat{t}_{1}, \hat{t}_{2}\right)+\sum_{n=1}^{\infty} \epsilon^{n} \hat{C}_{n}\left(\hat{r}, \hat{z}, \hat{t}_{0}, \hat{t}_{1}, \hat{t}_{2}\right), \\
\hat{C}_{s}(\hat{z}, \hat{t})=\hat{C}_{s 0}\left(\hat{z}, \hat{t}_{1}, \hat{t}_{2}\right)+\sum_{n=1}^{\infty} \epsilon^{n} \hat{C}_{s n}\left(\hat{z}, \hat{t}_{0}, \hat{t}_{1}, \hat{t}_{2}\right) .
\end{gathered}
$$

In these expansions, the leading-order terms are anticipated not depending on the oscillatory short time $\hat{t}_{0}$, and the higher order terms can only be purely oscillatory functions of the short time $\hat{t}_{0}$.

On assuming small-amplitude waves and multiple time scales, we may expand the velocities and the time derivative into powers of $\epsilon$ :

$$
\begin{gathered}
(\hat{u}, \hat{w})=\epsilon\left(\hat{u}_{1}, \hat{w}_{1}\right)+\sum_{n=2}^{\infty} \epsilon^{n}\left(\hat{u}_{n}, \hat{w}_{n}\right) \\
\partial / \partial \hat{t}=\partial / \partial \hat{t}_{0}+\epsilon \partial / \partial \hat{t}_{1}+\epsilon^{2} \partial / \partial \hat{t}_{2}
\end{gathered}
$$

Due to the oscillatory pressure gradient, the leading order velocity components can be further expanded into:

$$
\left(\hat{u}_{1}, \hat{w}_{1}\right)=\operatorname{Re}\left[(\hat{U}, \hat{W}) e^{i(\hat{t}-\hat{k} \hat{z})}\right]
$$

where $\hat{U}$ and $\hat{W}$, which have been obtained by Ma and $\mathrm{Ng}$ [16], are given by

$$
\begin{gathered}
\hat{U}=i \frac{\hat{k}^{2} \hat{r}}{2} p^{o}-\hat{k} J_{1}(\lambda \hat{r}) B_{1}, \\
\hat{W}=\hat{k} p^{o}+i \lambda J_{0}(\lambda \hat{r}) B_{1}
\end{gathered}
$$

where $\lambda=\alpha i^{3 / 2}$, in which $\alpha=a\left(\sigma / \nu_{f}\right)^{1 / 2}$ (where $\nu_{f}$ is the kinematic viscosity of fluid) is the dimensionless Womersley number representing the oscillation frequency, and $J_{n}$ is the Bessel function of the first kind of order $n$. The complex wavenumber $\hat{k}=\hat{k}_{r}+i \hat{k}_{i}$ is given by a dispersion equation:

$$
\hat{k}^{2}=-\frac{\alpha^{4} \lambda J_{0}(\lambda)}{M\left(\alpha^{2} \gamma i+\beta\right)\left[J_{1}(\lambda)-\lambda J_{0}(\lambda) / 2\right]\left[2\left(1-1 / \hat{b}^{2}\right)-\epsilon^{2} \alpha^{4} \ln (\hat{b}) /\left(\alpha^{2} \gamma i+\beta\right)+\chi\right]} .
$$

In this equation, $M=\rho_{w} / \rho_{f}$ is the ratio of wall density $\rho_{w}$ to fluid density, $\beta=$ $\epsilon^{2} a^{2} G_{w} / \rho_{w} \nu_{f}^{2}$ is a parameter representing the significance of the tube elasticity, in 
which $G_{w}$ is the shear modulus of the wall, $\gamma=\epsilon^{2} \nu_{w} / \nu_{f}$ is a ratio of the kinematic viscosity of the wall $\nu_{w}$ to that of the fluid, and

$$
\chi=\frac{\beta}{\left(\alpha^{2} \gamma i+\beta\right) S}\left(1-\frac{1}{\hat{b}^{2}}-\frac{j_{1}}{2}+\frac{j_{1}}{2 \hat{b}^{4}}+\frac{2}{j_{1}} \ln \frac{\hat{b} \sqrt{1-j_{1}}}{\sqrt{\hat{b}^{2}-j_{1}}}\right),
$$

and $j_{1}$ is governed by:

$$
\ln \left[\frac{\hat{b}^{2}\left(1-j_{1}\right)}{\hat{b}^{2}-j_{1}}\right]-\left(1-\hat{b}^{-2}\right) j_{1}=-2 S \hat{Q},
$$

in which $\hat{Q}=Q / G_{w}$ and $S$ are the initial steady fluid pressure and the longitudinal stretch ratio, respectively. For a constrained tethered tube, $\chi$ is zero in the absence of initial stresses. By the boundary conditions of motions, the complex constant $B_{1}$ in Eqs. (19) and (20) can be expressed as follows:

$$
B_{1}=\frac{i \hat{k} p^{o}}{\lambda J_{0}(\lambda)} .
$$

The fluid-wall interface displacement $\hat{\xi}$ and the radial velocity $\hat{U}$ are related by

$$
\hat{\xi}=-i \hat{U}^{(1)}=\frac{\hat{k}^{2} p^{o}}{2}+i \hat{k} J_{1}(\lambda) B_{1} .
$$

Successive orders of perturbation equations are obtainable by substituting Eqs. (14)(17) into Eqs. (11)-(13). These equations and their solutions are discussed below.

\section{$3 \quad$ Asymptotic analysis}

\subsection{Leading order}

For $O(1)$, Eqs. (11)-(13) give

$$
\begin{gathered}
0=\frac{1}{\hat{r}} \frac{\partial}{\partial \hat{r}}\left(\hat{r} \frac{\partial \hat{C}_{0}}{\partial \hat{r}}\right) \\
\frac{\partial \hat{C}_{0}}{\partial \hat{r}}=0, \quad \hat{r}=0 \\
-\frac{1}{\operatorname{Pe}} \frac{\partial \hat{C}_{0}}{\partial \hat{r}}=0=\hat{\kappa}\left(\hat{\omega} \hat{C}_{0}-\hat{C}_{s 0}\right), \quad \hat{r}=1 .
\end{gathered}
$$


From the equations above, one can easily get that the leading-order concentration $\hat{C}_{0}$ is independent of $\hat{r}$ :

$$
\hat{C}_{0}=\hat{C}_{0}\left(\hat{z}, \hat{t}_{1}, \hat{t}_{2}\right)
$$

and

$$
\hat{C}_{s 0}=\hat{\omega} \hat{C}_{0}
$$

which imply that the fluid phase of the substance is at local equilibrium with the wall phase at the leading order.

\subsection{First order}

At $O(\epsilon)$, Eqs. (11)-(13) give

$$
\begin{array}{cc}
\operatorname{Pe}\left[\frac{\partial \hat{C}_{0}}{\partial \hat{t}_{1}}+\frac{\partial \hat{C}_{1}}{\partial \hat{t}_{0}}+\operatorname{Re}\left[\hat{W} e^{i(\hat{t}-\hat{k} \hat{z})}\right] \frac{\partial \hat{C}_{0}}{\partial \hat{z}}\right]=\frac{1}{\hat{r}} \frac{\partial}{\partial \hat{r}}\left(\hat{r} \frac{\partial \hat{C}_{1}}{\partial \hat{r}}\right), \quad 0<\hat{r}<1, \\
\frac{\partial \hat{C}_{1}}{\partial \hat{r}}=0, \quad \hat{r}=0, \\
-\frac{1}{\operatorname{Pe}} \frac{\partial \hat{C}_{1}}{\partial \hat{r}}-\hat{\Gamma} \hat{C}_{0}=\frac{\partial \hat{C}_{s 0}}{\partial \hat{t}_{1}}+\frac{\partial \hat{C}_{s 1}}{\partial \hat{t}_{0}}=\hat{\kappa}\left(\hat{\omega} \hat{C}_{1}-\hat{C}_{s 1}\right), \quad \hat{r}=1 .
\end{array}
$$

Taking average of Eqs. (31)-(33) with respect to the fast time variable $\hat{t}_{0}$, we get

$$
\begin{gathered}
\operatorname{Pe} \frac{\partial \hat{C}_{0}}{\partial \hat{t}_{1}}=\frac{1}{\hat{r}} \frac{\partial}{\partial \hat{r}}\left(\hat{r} \frac{\partial \overline{\hat{C}}_{1}}{\partial \hat{r}}\right), \quad 0<\hat{r}<1, \\
\frac{\partial \hat{\hat{C}}_{1}}{\partial \hat{r}}=0, \quad \hat{r}=0, \\
-\frac{1}{\operatorname{Pe}} \frac{\partial \hat{C}_{1}}{\partial \hat{r}}-\hat{\Gamma} \hat{C}_{0}=\frac{\partial \hat{C}_{s 0}}{\partial \hat{t}_{1}}=\hat{\kappa}\left(\hat{\omega} \hat{C}_{1}-\overline{\hat{C}}_{s 1}\right), \quad \hat{r}=1,
\end{gathered}
$$

where the overbar denotes time average over one period. By further taking section average of Eq. (34) and using Eqs. (30), (35) and (36), we may get:

$$
\frac{\partial \hat{C}_{0}}{\partial \hat{t}_{1}}+\frac{2 \hat{\Gamma}}{R} \hat{C}_{0}=0
$$

where $R=1+2 \hat{\omega}$ is the retardation factor resulting from the reversible partitioning of the species into two phases. Eq. (37) is the leading-order effective transport equation describing the rate of change on the medium time-scale $T_{1}$, which is solely controlled 
by the irreversible wall absorption at a rate of $2 \hat{\Gamma} / R$. There is no net advective transport as the velocity at this scale is purely oscillatory with a zero time mean.

Now the expressions for the $O(\epsilon)$ concentrations can be found. Substituting Eq. (37) back into Eqs. (31) and (33) gives

$$
\operatorname{Pe}\left[\frac{\partial \hat{C}_{1}}{\partial \hat{t}_{0}}+\operatorname{Re}\left[\hat{W} e^{i(\hat{t}-\hat{k} \hat{z})}\right] \frac{\partial \hat{C}_{0}}{\partial \hat{z}}-\frac{2 \hat{\Gamma}}{R} \hat{C}_{0}\right]=\frac{1}{\hat{r}} \frac{\partial}{\partial \hat{r}}\left(\hat{r} \frac{\partial \hat{C}_{1}}{\partial \hat{r}}\right), \quad 0<\hat{r}<1,
$$

and

$$
-\frac{1}{\operatorname{Pe}} \frac{\partial \hat{C}_{1}}{\partial \hat{r}}=\frac{\partial \hat{C}_{s 1}}{\partial \hat{t}_{0}}+\frac{\hat{\Gamma}}{R} \hat{C}_{0}=\hat{\kappa}\left(\hat{\omega} \hat{C}_{1}-\hat{C}_{s 1}\right)+\hat{\Gamma} \hat{C}_{0}, \quad \hat{r}=1,
$$

where Eq. (30) has been used. Note that Eqs. (38) and (39) are basically the same as those when the tube is rigid, as has been deduced by $\mathrm{Ng}$ [17], only except for the expression of the leading-order axial velocity $\hat{W}$. The flexibility of the tube will influence the advection and dispersion coefficients of the mass transport in the next order, as will be deduced later.

According to Eqs. (34) and (36), one can express the first-order concentrations $\hat{C}_{1}$ and $\hat{C}_{s 1}$ as follows:

$$
\hat{C}_{1}=\operatorname{Re}\left[N(\hat{r}) e^{i(\hat{t}-\hat{k} \hat{z})}\right] \frac{\partial \hat{C}_{0}}{\partial \hat{z}}+M(\hat{r}) \hat{C}_{0}
$$

and

$$
\hat{C}_{s 1}=\operatorname{Re}\left[N_{s} e^{i(\hat{t}-\hat{k} \hat{z})}\right] \frac{\partial \hat{C}_{0}}{\partial \hat{z}}+M_{s} \hat{C}_{0}
$$

where $N(\hat{r}), N_{s}, M(\hat{r})$ and $M_{s}$ satisfy the boundary-value problems below.

On matching with the steady terms associated with $\hat{C}_{0}$, one can get the following equations for $M(\hat{r})$ and $M_{s}$ :

$$
\frac{1}{\hat{r}} \frac{d}{d \hat{r}}\left(\hat{r} \frac{d M}{d \hat{r}}\right)=-2 \mathrm{Pe} \frac{\hat{\Gamma}}{R}, \quad 0<\hat{r}<1,
$$

and

$$
-\frac{1}{\mathrm{Pe}} \frac{d M}{d \hat{r}}=\frac{\hat{\Gamma}}{R}=\hat{\kappa}\left(\hat{\omega} M-M_{s}\right)+\hat{\Gamma}, \quad \hat{r}=1 .
$$

Similarly, on matching with the oscillatory terms, one can obtain the following equations for $N(\hat{r})$ and $N_{s}$ :

$$
\frac{1}{\hat{r}} \frac{d}{d \hat{r}}\left(\hat{r} \frac{d N}{d \hat{r}}\right)-\operatorname{Pe}[i N(\hat{r})+\hat{W}]=0, \quad 0<\hat{r}<1,
$$

and

$$
-\frac{1}{\mathrm{Pe}} \frac{d N}{d \hat{r}}=i N_{s}=\hat{\kappa}\left(\hat{\omega} N-N_{s}\right), \quad \hat{r}=1 .
$$

Solutions to these boundary-value problems are presented in Section 4. 


\subsection{Second order}

At $O\left(\epsilon^{2}\right)$, Eqs. (11)-(13) give

$$
\begin{aligned}
\operatorname{Pe}\left[\frac{\partial \hat{C}_{0}}{\partial \hat{t}_{2}}+\frac{\partial \hat{C}_{1}}{\partial \hat{t}_{1}}+\frac{\partial \hat{C}_{2}}{\partial \hat{t}_{0}}+\frac{\partial \hat{w}_{1} \hat{C}_{1}}{\partial \hat{z}}+\frac{\partial \hat{r} \hat{u}_{1} \hat{C}_{1}}{\hat{r} \partial \hat{r}}+\hat{w}_{2} \frac{\partial \hat{C}_{0}}{\partial \hat{z}}\right]=\frac{1}{\hat{r}} \frac{\partial}{\partial \hat{r}}\left(\begin{array}{c}
\left.\hat{r} \frac{\partial \hat{C}_{2}}{\partial \hat{r}}\right)+\frac{\partial^{2} \hat{C}_{0}}{\partial \hat{z}^{2}} \\
0<\hat{r}<1, \quad(46)
\end{array}\right. \\
\frac{\partial \hat{C}_{2}}{\partial \hat{r}}=0, \quad \hat{r}=0 \\
-\frac{1}{\operatorname{Pe}}\left[\frac{\partial \hat{C}_{2}}{\partial \hat{r}}+\hat{\xi} \frac{\partial^{2} \hat{C}_{1}}{\partial \hat{r}^{2}}\right]-\hat{\Gamma} \hat{C}_{1}=\frac{\partial \hat{C}_{s 0}}{\partial \hat{t}_{2}}+\frac{\partial \hat{C}_{s 1}}{\partial \hat{t}_{1}}+\frac{\partial \hat{C}_{s 2}}{\partial \hat{t}_{0}} \\
=\hat{\kappa}\left(\hat{\omega} \hat{C}_{2}-\hat{C}_{s 2}\right)+\hat{\xi}\left(\omega \frac{\partial \hat{C}_{1}}{\partial \hat{r}}-\frac{\partial \hat{C}_{s 1}}{\partial \hat{r}}\right) \\
\hat{r}=1
\end{aligned}
$$

We first get for the following terms, on making use of Eqs. (37), (40) and (41),

$$
\begin{gathered}
\frac{\partial\left\langle\overline{\hat{C}}_{1}\right\rangle}{\partial \hat{t}_{1}}=-\frac{2 \hat{\Gamma}}{R}\langle M\rangle \hat{C}_{0}, \\
\frac{\partial \hat{\hat{C}}_{s 1}}{\partial \hat{t}_{1}}=-\frac{2 \hat{\Gamma}}{R} M_{s} \hat{C}_{0},
\end{gathered}
$$

and

$$
\frac{\partial\left\langle\overline{\hat{w}_{1} \hat{C}_{1}}\right\rangle}{\partial \hat{z}}=\frac{1}{2} \operatorname{Re}\left\langle\hat{W} N^{*}\right\rangle\left(\frac{\partial^{2} \hat{C}_{0}}{\partial \hat{z}^{2}}+2 \hat{k}_{i} \frac{\partial \hat{C}_{0}}{\partial \hat{z}}\right) e^{2 \hat{k}_{i} \hat{z}},
$$

where the asterisk denotes the complex conjugate, and the angle brackets denote averaging across the tube section. The successive temporal and sectional averaging of Eq. (46), subjected to the boundary conditions (47) and (48), will give the following effective transport equation:

$$
\frac{\partial \hat{C}_{0}}{\partial \hat{t}_{2}}+\frac{W_{O}}{R} \frac{\partial \hat{C}_{0}}{\partial \hat{z}}+\psi \frac{2 \hat{\Gamma}}{R} \hat{C}_{0}=\left(\frac{1}{\operatorname{Pe} R}+D_{E}\right) \frac{\partial^{2} \hat{C}_{0}}{\partial \hat{z}^{2}},
$$

where

$$
W_{O}=W_{L}+W_{D}
$$

is the advection velocity, and

$$
W_{L}=W_{E}+W_{S}
$$

in which

$$
W_{E}=\left\langle\widehat{\hat{w}}_{2}\right\rangle,
$$


is the section-averaged Eulerian steady streaming velocity,

$$
W_{S}=-\operatorname{Re}\left(i \hat{U}^{(1)} \hat{W}^{*(1)}\right) e^{2 \hat{k}_{i} \hat{z}}
$$

is an additional component of the advection velocity, and $\hat{k}_{i}$ is the imaginary part of the complex wavenumber $\hat{k}$.

$$
W_{D}=\left[\hat{k}_{i} \operatorname{Re}\left\langle\hat{W} N^{*}\right\rangle-\operatorname{Re}\left(\frac{\hat{\xi}}{\operatorname{Pe}} \frac{d N^{*(1)}}{d \hat{r}}\right)\right] e^{2 \hat{k}_{i} \hat{z}},
$$

is another additional velocity due to the wave damping as well as the wall reaction. $W_{D}$ can be absent, if the wave attenuation is ignored $\left(\hat{k}_{i}=0\right)$ and the wall is inert $\left(d N^{*(1)} / d \hat{r}=0\right)$. The coefficient

$$
\psi=-\frac{\langle M\rangle}{R}+M^{(1)}-\frac{2 M_{s}}{R},
$$

is a higher-order correction factor to the decay rate, and

$$
D_{E}=-\frac{1}{2 R} \operatorname{Re}\left\langle\hat{W} N^{*}\right\rangle e^{2 \hat{k}_{i} \hat{z}}
$$

is a dispersion coefficient due to the axial oscillatory fluid motion. Eq. (52) is an effective transport equation describing the slow time rate of change, which is controlled by advection, leading-order decay, diffusion and more important, dispersion. The advection and dispersion coefficients above are normalized by $\epsilon^{2} L \sigma$ and $\epsilon^{2} L^{2} \sigma$, respectively. It is remarkable that $\psi$ is solely caused by the irreversible wall absorption and the dispersion coefficient $D_{E}$ is affected only by the reversible phase exchange. The effects of the wall absorption on the dispersion can be found at a higher order, but is omitted in the present study. It is only a higher-order correction to $D_{E}$ with a much smaller magnitude.

\section{Effective coefficients}

\subsection{Correction factor $\psi$}

First, $M(\hat{r})$ and $M_{s}$ can be readily found by solving Eqs. (42) and (43):

$$
M(\hat{r})=M(0)-\frac{\mathrm{Pe} \hat{\Gamma} \hat{r}^{2}}{2 R}
$$


and

$$
M_{s}=\hat{\omega} M(0)+\frac{2 \hat{\omega} \hat{\Gamma}}{R}\left(\frac{1}{\hat{\kappa}}-\frac{P e}{4}\right),
$$

where $M(0)$ is an unknown constant, but will have no effect on the evaluation of $\psi$ as shown in Eq. (62) below. After some algebra upon substitution of $M$ and $M_{s}$, the correction factor $\psi$ can be deduced as follows:

$$
\psi=-\frac{2 \mathrm{Pe} \hat{\Gamma}}{R}\left[\frac{1}{8 R}+\frac{1}{\hat{\kappa} \mathrm{Pe}}\left(1-\frac{1}{R}\right)\right] .
$$

\subsection{Advection velocity $W_{O}$}

We first show that the additional advection velocity component $W_{S}$ is actually equal to the section-averaged Stokes drift. For the present axisymmetric problem, Stokes drift [21] is given by:

$$
\begin{aligned}
\overline{\left(\int^{\hat{t}} \hat{w}_{1} d \hat{t}\right) \frac{d \hat{w}_{1}}{d \hat{z}}}+\overline{\left(\int^{\hat{t}} \hat{u}_{1} d \hat{t}\right) \frac{d \hat{w}_{1}}{d \hat{r}}} & =-i \hat{w}_{1}\left(\frac{\partial \hat{u}_{1}}{\partial \hat{r}}+\frac{\hat{u}_{1}}{\hat{r}}\right)-i \hat{u}_{1} \frac{\partial \hat{w}_{1}}{\partial \hat{r}} \\
& =-\frac{\operatorname{Re}}{2}\left[i \frac{\partial\left(\hat{r} \hat{U} \hat{W}^{*}\right)}{\hat{r} \partial \hat{r}}\right] e^{2 \hat{k}_{i} \hat{z}}
\end{aligned}
$$

whose cross-section average is clearly equal to $W_{S}$ :

$$
-\frac{\operatorname{Re}}{2}\left\langle i \frac{\partial\left(\hat{r} \hat{U} \hat{W}^{*}\right)}{\hat{r} \partial \hat{r}}\right\rangle e^{2 \hat{k}_{i} \hat{z}}=-\operatorname{Re}\left(i \hat{U}^{(1)} \hat{W}^{*(1)}\right) e^{2 \hat{k}_{i} \hat{z}}
$$

Therefore, in Eq. (54), the advection velocity $W_{L}$ is the section-averaged sum of the Eulerian steady streaming velocity and the Stokes drift, which amounts to the sectionaveraged Lagrangian drift or steady streaming of individual fluid particles. As shown by $\mathrm{Ma}$ and $\mathrm{Ng}$ [16], the net Lagrangian time-mean flux across a section is identically equal to zero, $W_{L}=0$, when the time-mean motion of the wall dies out.

The additional velocity $W_{D}$ can be found after solving for $N$. The boundary conditions in Eq. (45) for $N(\hat{r})$ can be rewritten as

$$
\frac{1}{\mathrm{Pe}} \frac{d N}{d \hat{r}}=\tau N, \quad \hat{r}=1
$$

where

$$
\tau=-\frac{\hat{\kappa} \hat{\omega} i}{i+\hat{\kappa}} .
$$


With the expression of $\hat{W}$ given in Eq. (20), and the boundary condition above, Eq. (44) has the following solution:

$$
N(\hat{r})=\hat{k} p^{o} i-\frac{\operatorname{Pe} \lambda J_{0}(\lambda \hat{r}) B_{1}}{\operatorname{Pe}-\alpha^{2}}+H J_{0}(\eta \hat{r}),
$$

where

$$
\eta=\mathrm{Pe}^{1 / 2} i^{3 / 2}=\mathrm{Sc}^{1 / 2} \lambda
$$

and

$$
H=\frac{\operatorname{Pe} \lambda B_{1}\left[\lambda J_{1}(\lambda)+\tau \operatorname{Pe} J_{0}(\lambda)\right]}{\left(\operatorname{Pe}-\alpha^{2}\right)\left[\eta J_{1}(\eta)+\tau \operatorname{Pe} J_{0}(\eta)\right]}-\frac{\operatorname{Pe} \tau \hat{k} p^{o} i}{\eta J_{1}(\eta)+\tau \operatorname{Pe} J_{0}(\eta)},
$$

in which $\mathrm{Sc}=\nu_{f} / D$ is the Schmidt number. After some algebra upon substituting Eqs. (25) and (67) into Eq. (57), one may find that

$$
\begin{aligned}
W_{D}= & 2 \hat{k}_{i} e^{2 \hat{k}_{i} \hat{z}} \operatorname{Re}\left[\frac{\hat{k} p^{o} H^{*} J_{1}\left(\eta^{*}\right)}{\eta^{*}}-\frac{\hat{k} p^{o} B_{1}^{*} \alpha^{2} J_{1}\left(\lambda^{*}\right)}{\mathrm{Pe}-\alpha^{2}}\right. \\
& \left.-\frac{\lambda B_{1} H^{*}\left[\lambda J_{0}\left(\eta^{*}\right) J_{1}(\lambda)-\eta^{*} J_{1}\left(\eta^{*}\right) J_{0}(\lambda)\right]}{\mathrm{Pe}+\alpha^{2}}\right] \\
& -e^{2 \hat{k}_{i} \hat{z}} \operatorname{Re}\left[\left(\frac{\hat{k}^{2} p^{o}}{2}+i \hat{k} J_{1}(\lambda) B_{1}\right)\left(\frac{\lambda^{* 2} J_{1}\left(\lambda^{*}\right) B_{1}^{*}}{\mathrm{Pe}-\alpha^{2}}-\frac{H^{*} \eta^{*} J_{1}\left(\eta^{*}\right)}{\mathrm{Pe}}\right)\right] .
\end{aligned}
$$

\subsection{Dispersion coefficient $D_{E}$}

With Eqs. (44) and (65), Eq. (59) can be manipulated to become

$$
D_{E}=\frac{e^{2 \hat{k}_{i} \hat{z}}}{2 \operatorname{Pe} R}\left\langle\left|\frac{d N}{d \hat{r}}\right|^{2}\right\rangle-\frac{e^{2 \hat{k}_{i} \hat{z}}}{R} \operatorname{Re}(\tau)|N|^{2} .
$$

On the substitution of Eq. (67) into Eq. (59), an explicit expression for $D_{E}$ can be obtained as follows:

$$
\begin{aligned}
D_{E}= & -\frac{e^{2 \hat{k}_{i} \hat{z}}}{R} \operatorname{Re}\left\{\frac{\hat{k} p^{o} H^{*} J_{1}\left(\eta^{*}\right)}{\eta^{*}}-\frac{\hat{k} p^{o} B_{1}^{*} \alpha^{2} J_{1}\left(\lambda^{*}\right)}{\operatorname{Pe}-\alpha^{2}}\right. \\
& \left.-\frac{\lambda B_{1} H^{*}\left[\lambda J_{0}\left(\eta^{*}\right) J_{1}(\lambda)-\eta^{*} J_{1}\left(\eta^{*}\right) J_{0}(\lambda)\right]}{\operatorname{Pe}+\alpha^{2}}\right\} .
\end{aligned}
$$

This dispersion coefficient is the one for oscillatory flow in a flexible tube subject to kinetic phase exchange between the fluid and the wall. By using L'Hospital's rule, one may show that the above expression has the following finite limit for $\mathrm{Pe}=\alpha^{2}$ or 
$\mathrm{Sc}=1:$

$$
\begin{aligned}
\lim _{S c \rightarrow 1} D_{E}= & -\frac{e^{2 \hat{k}_{i} \hat{z}}}{2 R \alpha^{2}} \operatorname{Re}\left\{\lambda ^ { - 1 } [ \lambda J _ { 1 } ( \lambda ) + \tau \alpha ^ { 2 } J _ { 0 } ( \lambda ) ] ^ { - 1 } \left[\hat{k}^{*} p^{o *} B_{1} \lambda^{2} \tau \alpha^{4}\left(J_{0}^{2}(\lambda)+J_{1}^{2}(\lambda)\right)\right.\right. \\
& +|\lambda|^{2} B_{1}^{*} \operatorname{Im}\left[\lambda J_{0}\left(\lambda^{*}\right) J_{1}(\lambda)\right]\left[2 \alpha^{2} \tau \hat{k} p^{o}-i B_{1} J_{0}(\lambda) \lambda^{3}+i \alpha^{2} B_{1} \tau J_{1}(\lambda) \lambda^{2}\right] \\
& \left.\left.-2 i \tau|\hat{k}|^{2}\left|p^{o}\right|^{2} J_{1}(\lambda) \alpha^{4}\right]\right\}-\frac{|\lambda|^{4}\left|B_{1}\right|^{2}\left|J_{1}(\lambda)\right|^{2} e^{2 \hat{k}_{i} \hat{z}}}{4 R \alpha^{2}}
\end{aligned}
$$

where Im stands for the imaginary part. In the limit of a perfectly rigid tube, or $\beta \rightarrow \infty$, one can find that $D_{E}$ in Eq. (72) can reduce to the dispersion coefficient obtained previously by $\mathrm{Ng}$ [17], who studied the dispersion of a chemical species in steady and oscillatory flow though a rigid reactive tube.

\section{Comparison with DG91 [13]}

To check our theory, we first compare results with those obtained by DG91 [13], who studied mass transport in pulmonary airways during high-frequency ventilation (HFV) without wall reactions and initial stresses. Following their work, the physical values are chosen as follows: $a=9 \times 10^{-3} \mathrm{~m}, \hat{b}=b / a=1.056$ is the ratio of the tube thickness to the inner tube radius, $\epsilon=0.1, \rho_{f}=1.14 \mathrm{~kg} / \mathrm{m}^{3}, \rho_{w}=9.97 \times 10^{2} \mathrm{~kg} / \mathrm{m}^{3}$ $(M=874.56), \nu_{f}=1.5 \times 10^{-5} \mathrm{~m}^{2} / \mathrm{s}, \nu_{w}=1.125 \times 10^{-4} \mathrm{~m}^{2} / \mathrm{s}, G_{w}=138.5 \mathrm{~Pa}$ for normal tracheae $(\beta=500, \gamma=0.075), \mathrm{Sc}=0.882$ results $\mathrm{Pe}=0.882 \alpha^{2}$. In HFV, it requires a fixed leading-order tidal volume $V_{T}$ at a given axial position, say $Z=0$, in one half-cycle, and $d=V_{T} / \pi a^{2}$ is the stroke length. Following DG91 [13], the dimensionless flow-rate can be expressed by:

$$
\int_{0}^{1} \hat{W} \hat{r} d \hat{r}=A / 4
$$

where $A=d / a$ is a non-dimensional amplitude parameter, relating the stroke length to the undisturbed inner tube radius. In this study, $A$ is fixed at 5 .

Since the steady state is considered, $\hat{C}_{0}$ can be assumed to be independent of time,

such that $\hat{C}_{0}=\hat{C}_{0}(\hat{z})$. For the steady transport through an inert tube, the effective transport equation (52) becomes:

$$
W_{D C} e^{2 \hat{k}_{i} \hat{z}} \frac{\partial \hat{C}_{0}}{\partial \hat{z}}=\left(\frac{1}{\mathrm{Pe}}+D_{E C} e^{2 \hat{k}_{i} \hat{z}}\right) \frac{\partial^{2} \hat{C}_{0}}{\partial \hat{z}^{2}}
$$


where

$$
D_{E C}=\operatorname{Re}\left\{\frac{\lambda\left|B_{1}\right|^{2} \mathrm{Sc}^{1 / 2} J_{1}\left(\lambda^{*}\right)\left[J_{0}\left(\eta^{*}\right) J_{1}(\lambda)-i S c^{1 / 2} J_{1}\left(\eta^{*}\right) J_{0}(\lambda)\right]}{\left(\mathrm{Sc}^{2}-1\right) J_{1}\left(\eta^{*}\right)}\right\}
$$

and

$$
W_{D C}=-2 \hat{k}_{i} D_{E C}
$$

are the dispersion and advection coefficients, respectively $\left(\hat{k}_{i}<0\right)$. It is remarkable that the transport equation (75) for the leading-order concentration $\hat{C}_{0}$ is essentially the same as Eq. (6.9) of DG91 [13], but with the dependence on the flow, e.g., wavenumber and axial velocity, now clearly visible in the coefficients. By virtue of the homogenization technique, we have deduced explicit expressions for the two effective coefficients. The transport mechanisms are now more clearly described by the expressions, and the coefficients are much easier to evaluate than those in DG91 [13].

Let us further prescribe steady boundary conditions for the concentrations at the two axial ends of the tube:

$$
\hat{C}_{0}(0)=1, \quad \text { and } \quad \hat{C}_{0}(1)=0 \text {. }
$$

Based on these boundary conditions, Eq. (75) can be readily solved to give

$$
\hat{C}_{0}=-\frac{2 \hat{k}_{i} \hat{z}+\ln \left(\frac{1+\mathrm{Pe} D_{E C}}{1+\operatorname{Pe} D_{E C} e^{2 \hat{k}_{i} \hat{z}}}\right)}{2 \hat{k}_{i}+\ln \left(\frac{1+\operatorname{Pe} D_{E C}}{1+\operatorname{Pe} D_{E C} e^{2 \hat{k}_{i}}}\right)}+1,
$$

The dimensionless steady rate of longitudinal mass transport across the cross-section, averaged over one period, is given by

$$
\hat{m}=2 \pi \int_{0}^{1+\epsilon \hat{\xi}}\left(\hat{w} \hat{C}-\frac{\epsilon^{2}}{\operatorname{Pe}} \frac{\partial \hat{C}}{\partial \hat{z}}\right) \hat{r} d \hat{r}
$$

where the dimensional steady transport rate $m$ has been scaled by $\hat{m}=m /\left(L \sigma a^{2} C^{*}\right)=$ $m /\left(\epsilon^{-1} \alpha^{2} a \nu_{f} C^{*}\right)$. On substituting the asymptotic expansions in Eqs. (14)-(16) into Eq. (80), for an inert tube, $\hat{m}$ can be expressed by

$$
\begin{aligned}
\hat{m} & =2 \pi \epsilon^{2}\left\{\int_{0}^{1}\left[\overline{\hat{w}_{1} \hat{C}_{1}}+\overline{\hat{w}_{2} \hat{C}_{0}}-\frac{1}{\mathrm{Pe}} \frac{d \hat{C}_{0}}{d \hat{z}}\right] \hat{r} d \hat{r}+\left.\overline{\hat{\xi} \hat{w}_{1}} \hat{C}_{0}\right|_{\hat{r}=1}\right\} \\
& =-\pi \epsilon^{2}\left\{\left[\frac{\operatorname{Re}\left\langle W N^{*}\right\rangle}{2} e^{2 \hat{k}_{i} \hat{z}}+\frac{1}{\mathrm{Pe}}\right] \frac{\partial \hat{C}_{0}}{\partial \hat{z}}+\left[\left\langle\hat{w}_{2}\right\rangle-\operatorname{Re}\left(i \hat{U}^{(1)} \hat{W}^{*(1)}\right) e^{2 \hat{k}_{i} \hat{z}}\right] \hat{C}_{0}\right\} \\
& =-\pi \epsilon^{2}\left\{\left[D_{E}+\mathrm{Pe}^{-1}\right] \frac{\partial \hat{C}_{0}}{\partial \hat{z}}+W_{L} \hat{C}_{0}\right\} .
\end{aligned}
$$


By the condition of zero section-averaged steady streaming flux $\left(W_{L}=0\right)$ and Eq. (79), $\hat{m}$ can be readily manipulated to give

$$
\hat{m}=\frac{\pi \epsilon^{2}}{\operatorname{Pe}\left[1+\left(2 \hat{k}_{i}\right)^{-1} \ln \left(\frac{1+\operatorname{Pe} D_{E C}}{1+\operatorname{Pe} D_{E C} e^{\hat{k}_{i}}}\right)\right]} .
$$

The expression above for steady axial transport does not depend on the axial coordinate $\hat{z}$, which is required by the conservation of mass for an inert species.

The dimensionless steady rate of transport based on DG91's [13] viscoelastic wall model can be re-deduced to be in a form more concise than that expressed in their paper:

$$
m^{\prime}=\frac{\pi \epsilon}{\operatorname{Sc}\left[1+\left(2 \hat{k}_{i}^{\prime}\right)^{-1} \ln \left(\frac{1+D_{E C}^{\prime}}{1+D_{E C}^{\prime} e^{2 \hat{k}_{i}^{\prime}}}\right)\right]}
$$

where

$$
\begin{gathered}
D_{E C}^{\prime}=\int_{0}^{1}\left|\frac{d \hat{N}^{\prime}(\hat{r})}{d \hat{r}}\right|^{2} \hat{r} d \hat{r}, \\
N^{\prime}(\hat{r})=B J_{0}\left(\lambda \mathrm{Sc}^{1 / 2} \hat{r}\right)+Z_{1}(\hat{r}) J_{0}\left(\lambda \mathrm{Sc}^{1 / 2} \hat{r}\right)+Z_{2}(\hat{r}) Y_{0}\left(\lambda \mathrm{Sc}^{1 / 2} \hat{r}\right), \\
Z_{1}(\hat{r})=-\frac{1}{2} \pi \mathrm{Sc} \int_{0}^{\hat{r}} W_{0}(s) Y_{0}\left(\lambda \mathrm{Sc}^{1 / 2} s\right) s d s, \\
Z_{2}(\hat{r})=-\frac{1}{2} \pi \mathrm{Sc} \int_{0}^{\hat{r}} W_{0}(s) J_{0}\left(\lambda \mathrm{Sc}^{1 / 2} s\right) s d s, \\
W_{0}(s)=\frac{A \alpha^{2}}{4} \frac{J_{0}(\lambda)}{J_{2}(\lambda)}\left[\frac{J_{0}(\lambda s)}{J_{0}(\lambda)}-1\right], \\
R_{0}=1+\frac{2 J_{0}(\lambda)}{J_{2}(\lambda)} \frac{\hat{m}\left[R_{0}^{2} \alpha^{4}-i R_{0}^{2}(\tilde{\gamma}) \alpha^{2}-\tilde{\kappa} / 0.75\right]}{2 a}, \quad \tilde{M}=\frac{\epsilon^{2} \rho_{w}(b-a)}{\rho_{f} a}, \quad \tilde{\gamma}=\frac{g a^{2}}{\nu_{f}}, \quad \lambda=\alpha i^{3 / 2}, \quad \tilde{\kappa}=\frac{E a^{2}}{\rho_{w} \nu_{f}^{2}},
\end{gathered}
$$

where $E=3 G_{w}$ is Young's modulus for an incompressible tube and $g$ is the wall damping. The complex constant $B$ is determined by the no-flux condition: $d N^{\prime} / d \hat{r}=$ 0. $Y_{0}$ is the zeroth-order Bessel function of the second kind, and $\hat{k}^{\prime}$ is the complex wavenumber in DG91's [13] model. Since $m^{\prime}$ has been scaled by $a \nu_{f} C^{*}$ in DG91 [13], $\hat{m}$ here should be written as $\hat{m}^{\prime}=\epsilon^{-1} \alpha^{2} \hat{m}$ for possible comparison with $m^{\prime}$. As was assumed by DG91 [13], the initial stresses are ignored in this particular example.

In DG91's [13] model, the viscous damping is accounted for by a dissipation term added directly to the inertia alongside the acceleration terms in the momentum equations deduced previously by Atabek and Lew [22]. In principle, the viscous damping 
term should better be incorporated into the stress terms through a viscoelastic Voigt model. With the comparison of the two models, one finds that

$$
\gamma=\epsilon^{2} \tilde{\gamma} / 4
$$

which relates the wall damping $g$ (used in DG91 [13]) with the viscous coefficient $\nu_{w}$ (used in the present work), by which results of the present viscoelastic model can be compared with those presented in DG91 [13].

Fig. 1 shows a comparison of the steady mass transport rate and phase lag between the volume flow rate and wall response on the wall-fluid interface $\hat{r}=1$, as functions of the Womersley number $\alpha$ and the elasticity $\beta$, where the wall viscosity $\gamma=0.075$, between the present model and DG91 [13]. As clearly seen in the figure, the present results for gas transport are essentially in good agreement with those by DG91 [13]. The close agreement suggests that, as far as a thin-walled tube is considered $(\hat{b}<1.1)$, our dispersion model is as good as DG91's [13] model when applied to gas transport in pulmonary airways during HFV. Looking into Eqs. (71), (82), (83), and (84), one can find that the formulas of the two models for the transport rate are basically the same, except for the expressions for $N(\hat{r})$ and $\hat{N}^{\prime}(\hat{r})$, which are solely determined by the axial velocity. The differences between the two models, giving rise to different velocity fields, have been discussed in detail previously by $\mathrm{Ma}$ and $\mathrm{Ng}$ [16]. The disparities stem from some basic differences between the two models in handling the radial wall displacement. As was pointed out by Sera et al. [23], and Montaudon et al. [24], the mean thickness of pulmonary airways is usually larger than $15 \%$ of the lumen radius. Therefore, our thick-wall model for describing the gas transport in flexible airways is more general than DG91's [13] thin-wall model, which is good only for relatively small tube thickness $(\hat{b}<1.1)$.

Fig. 1(a) shows that for the same frequency, the transport rate increases as the wall stiffness increases. The curve for $\beta=500000$, practically a rigid wall, serves as the upper bound (i.e., the maximum possible) for the transport rate. Along this upper bound, the transport rate increases monotonically with the frequency. At a fixed frequency, the transport rate will in general get smaller as the tube becomes softer. For elasticity as small as, say $\beta=5$ and 50 , the relation between transport rate and frequency is non-monotonic; the transport rate can reach a local maximum at a certain frequency, which is termed the optimal frequency. When the optimal 
frequency is exceeded, the transport rate can drop dramatically to a low value. The optimal frequency was also discussed by DG91 [13]. An important conclusion drawn by them is that the optimal frequency for maximum transport rate is close to the frequency for the minimum phase angle of the interface displacement, suggesting that the interfacial phase lag is correlated to the mass transport rate. When examining the two frequencies within the working ranges of $\operatorname{HFV}(\alpha \leq 15)$ against the wall elasticity as illustrated in Fig. 2, we find that the two frequencies are in a nearly constant ratio close to unity. The two frequencies increase monotonically as $\beta$ increases and the ratio of the optimal frequency to the frequency for minimum interfacial phase angle falls within the range of $1.15-1.2$. The ratio tends to increase only slightly as the elasticity increases. Hence, the interfacial phase angle is a factor that can be used to gauge the gas transport rate in pulmonary airways during high-frequency ventilation; the optimal frequency ratio 1.15-1.2 offers a good reference for practical design.

DG91 [13] suggested that it is the convective term $\overline{\hat{w}_{1} \hat{C}_{1}} \hat{r}$ in Eq. (81) (amounting to the dispersion coefficient) that is responsible for the local maxima of the low- $\beta$ curves in Fig. 1(a). Having deduced a much more explicit expression (82) for the transport rate, we are here in a clearer position to identify more exactly the cause of these local peaks. As in Eq. (82), $\hat{m}$ is governed by the effective dispersion coefficient $D_{E C}$ and the imaginary part of the wavenumber $\hat{k}_{i}$ (representing the spatial decay rate). These coefficients are plotted respectively in Fig. 3(a,b) against the Womersley number $\alpha$ for various values of the elasticity $\beta$, where $\gamma=0.075$. It is interesting to find that $D_{E C}$ is a function of the frequency parameter $\alpha$, but not of the elasticity parameter $\beta$. Therefore, instead of the dispersion coefficient, it is more because of the local sharp decrease of $\hat{k}_{i}$ for small $\beta$, say $\beta<50$, that is responsible for the local peaks (followed by sharp decline) of the mass transport rate shown in Fig. 1(a). The imaginary part of the wavenumber $\hat{k}_{i}$ is always negative, and its magnitude stands for the rate of spatial decay of the waves along the tube. The local minimum (or maximum in terms of magnitude) of $\hat{k}_{i}$ shown in Fig. 3(b) corresponds to the occurrence of resonance $[13,16]$, during which the wall motion will be dramatically enhanced in magnitude. As the wall moves with a larger amplitude in the radial direction, the fluid will be forced to move more in this direction as well. As a result, the axial transport will be reduced, as has been discussed in DG91 [13]. This explains the sudden decline, leaving a local peak behind, of the transport rate for an elastic 
tube upon the occurrence of resonance.

That $D_{E C}$ is independent of the elasticity $\beta$ (in fact, independent of all the wall mechanical properties) can be explained as follows. Substituting Eqs. (20) and (24) into Eq. (74), one can obtain relations for $\hat{k} p^{o}$ and $B_{1}$ in terms of the stroke length $A$, which is fixed by the condition of volume-cycling. By further making use of Eq. (69), an alternative expression for the effective dispersion coefficient $D_{E}$ in Eq. (72) can be obtained as below:

$$
\begin{aligned}
D_{E}=D_{E C} e^{2 \hat{k}_{i} \hat{z}}= & \frac{A^{2} e^{2 \hat{k}_{i} \hat{z}}}{16\left|\frac{1}{2}-\frac{J_{1}(\lambda)}{\lambda J_{0}(\lambda)}\right|^{2}(\mathrm{Sc}-1) R} \operatorname{Re}\left\{i \frac{J_{1}(\lambda)-\mathrm{Sc}^{1 / 2} J_{1}(\eta) H^{\prime}}{\lambda J_{0}(\lambda)}\right. \\
& \left.-i \frac{H^{*} \operatorname{Sc}\left[\lambda J_{0}\left(\eta^{*}\right) J_{1}(\lambda)-\eta^{*} J_{1}\left(\eta^{*}\right) J_{0}(\lambda)\right]}{(\mathrm{Sc}+1) \lambda^{2}\left|J_{0}(\lambda)\right|^{2}}\right\},
\end{aligned}
$$

where

$$
H^{\prime}=\frac{\lambda J_{1}(\lambda)+\alpha^{2} \tau J_{0}(\lambda)}{\eta J_{1}(\eta)+\tau \operatorname{Pe} J_{0}(\eta)} .
$$

From this, one can see that the dispersion coefficient $D_{E C}$ (which is $D_{E}$ without the axial decay factor) is entirely independent of any of the wall parameters (elasticity $\beta$, viscosity $\gamma$, initial stresses $\hat{Q}$ and $S$, and the wall thickness $\hat{b}$ ). In fact, $D_{E C}$ in Eq. (92) can be shown to be the same coefficient as that in Eq. (4.39) of $\mathrm{Ng}$ [17], which is for volume-cycled oscillatory flow through a rigid tube. The dispersion coefficient will, however, be no longer independent of the wall mechanical properties when the tube is untethered.

\section{Numerical discussions on dispersion through a reactive wall}

Let us further examine the gas transport mechanism due to oscillatory flow through a reactive wall. We shall use the same physical values for a human trachea as those used by DG91 [13], except that a more general wall thickness is chosen: $\hat{b}=1.15$. Since the dispersion coefficient does not depend on the wall properties for a tethered tube as discussed above, we here do not study the effects of the Schmidt number Sc, the frequency parameter $\alpha$, the reversible reaction rate $\hat{\kappa}$ and the partition coefficient $\hat{\omega}$, as they have already been discussed previously by $\mathrm{Ng}$ [17]. Here, we first examine 
how the advection coefficient $W_{D}$ in Eq. (70) will vary with the wall properties. In order to pull out the term $e^{2 \hat{k}_{i} \hat{z}}$ from Eqs. (70) and (72), $W_{D}$ and $D_{E}$ can be written as

$$
\left(W_{D}, D_{E}\right)=\left(W_{D C}, D_{E C}\right) e^{2 \hat{k}_{i} \hat{z}},
$$

where $W_{D C}$ and $D_{E C}$ are independent of $\hat{z}$. Furthermore, an alternative expression for the reversible reaction rate $\hat{\kappa}$ in Eq. (66) can be written as

$$
\hat{\kappa}=\frac{\mathrm{Da}}{\alpha^{2} \mathrm{Sc}}
$$

where $\mathrm{Da}=\kappa a^{2} / D=O(1)$ is Damköhler number, representing the significance of the kinetics of the phase exchange. This redefinition will remove the dependence on frequency from the normalization. Fig. 4 shows the influences of the initial mean pressure $q=\left(a^{4} / \rho_{f} L^{2} \nu_{f}^{2}\right) Q=M \beta \hat{Q}$ and the longitudinal stretch ratio $S$ on the advection velocity $W_{D C}$. This redefinition will also remove the dependence on frequency from the normalization. For a normal trachea during HFV, $S \simeq 1.3$ [25], but the initial mean pressure is so tiny that DG91 [13] totally neglected it in their calculations. To illustrate the effects of initial stresses, the following ranges of values will be considered in our calculations: $q=0-2000$ and $S=1-1.4$. Fig. 4 shows the advection velocity $W_{D C}$ as a function of the frequency, initial mean pressure and stretch ratio, $\alpha, q$ and $S$, respectively, for a normal reactive wall. From Fig. 4(a), we find that the mean pressure enhances the advection velocity when the frequency is smaller than the resonance frequency, but the advection velocity is reduced by increasing the mean pressure if the frequency becomes much larger $(\alpha>12)$. In contrast, as shown in Fig. 4(b), the stretch ratio $S$ has no appreciable effect on the advection velocity. We have also checked other cases for different wall elasticity. The effects of $q$ and $S$ on the advection velocity is in general the same as those shown in Fig. 4(a) and (b). Hence, we may conclude that the initial mean pressure affects the advection velocity more appreciably than the stretch ratio.

Let us here consider a wall with only the reversible reaction, i.e., the absorption is zero $\hat{\Gamma}=0$, such that we can obtain an analytical solution of the leading-order concentration $\hat{C}_{0}$. Substitution of Eq. (94) into Eq. (52) with steady end concentrations (78), the leading-order concentration gradient along the $\hat{z}$-axis can be obtained as follows:

$$
\frac{\partial \hat{C}_{0}}{\partial \hat{z}}=-\frac{F(\hat{z})}{\int_{0}^{1} F(s) d s}
$$


where

$$
F(s)=\left(R D_{E C} e^{2 \hat{k}_{i} s}+\mathrm{Pe}^{-1}\right)^{\frac{W_{D C}}{2 \hat{k}_{i} R D_{E C}}} .
$$

Substitution of Eq. (96) into Eq. (81), the steady mass transport rate $\hat{m}^{\prime}$ for a reactive wall is

$$
\hat{m}^{\prime}=\pi \epsilon \alpha^{2} \frac{\left(R D_{E C} e^{2 \hat{k}_{i} s}+\mathrm{Pe}^{-1}\right) F(\hat{z})}{\int_{0}^{1} F(s) d s} .
$$

The expression above for $\hat{m}^{\prime}$ depends on the axial coordinate $\hat{z}$. Therefore, we should consider the average of $\hat{m}^{\prime}$ along the tube length:

$$
\overline{\hat{m}^{\prime}}=\int_{0}^{1} \hat{m}^{\prime} d \hat{z},
$$

which represents the axial-averaged steady transport rate of the solute through the flexible tube. To enable one to determine whether the effect of the parameters is to increase or to decrease the transport rate, we show in Fig. 5 how $\overline{\hat{m}^{\prime}}$ varies with the frequency $\alpha$ for several values of Sc, phase partition coefficient $\hat{\omega}$, Damköhler number Da and initial mean pressure $q$, respectively, where $\beta=50$ and $\gamma=0.075$. First, as shown in Fig. 5(a), with decreasing Sc (e.g., by increasing the molecular diffusivity), the magnitude of $\overline{\hat{m}^{\prime}}$ will be increased. Second, the phase partitioning and exchange rate have similar effects on the steady transport rate as illustrated in Fig. 5(b) and (c). When $\hat{\omega}$ or Da increases, the transport rate will be enhanced monotonically due to the combined effects of $D_{E C}, W_{D C}$ and $\hat{k}_{i}$, as given in Eq. (98). In physical terms, it means that an increase in the extent of wall retention or a faster rate of exchange between the wall and fluid phases will give rise to a larger quantity of the fluid phase being carried by the flow per unit time, amounting to a larger rate of mass transport through the tube. We have also checked other cases with different values of Sc and elasticity $\beta$, the dependence of the transport rate on the frequency is qualitatively the same as those shown in Fig. 5(b) and (c). It is also found that the transport rate will be independent of $\hat{\omega}$ and $\mathrm{Da}$ when the slow-oscillation limit is approached $(\alpha \rightarrow 0)$. Third, in Fig. 5(d), a higher value of $q$ may lead to smaller $\overline{\hat{m}^{\prime}}$, but the effect is significant only when the frequency $\alpha$ is not too small $(\alpha>7)$. Also, the stretch ratio $S$ tends to have little effect on the transport rate in a manner similar to that found in Fig. 4(b). By and large, the steady transport rate is affected to a greater extent by the initial mean pressure than by the initial stretch. Fourth, the axial-averaged transport rate can also reach a peak value at a particular frequency 
which becomes smaller for smaller Sc, larger phase partitioning, exchange rate and initial pressure, respectively.

\section{Concluding remarks}

By the multiple-scale method of homogenization, we have derived an effective transport equation (52) governing the advection and dispersion of a solute during volumecycled oscillatory flow in a prestressed and viscoelastic tube under the influences of kinetic phase exchange with the wall and first-order absorption into the wall. Because of wall viscous damping, all transport coefficients contain an exponential factor for axial decay along the tube. The advection velocity $W_{O}$ is composed of two parts: one part is related to the section-averaged steady streaming velocity, which is equal to zero when the time-mean motion of the wall dies out; another part is related to the wave damping and the wall reaction. The dispersion coefficient given in Eq. (72) is a function of the stroke length, the Womersley number, the Péclet number, and the reversible phase exchange. The particular case for an inert tube has been derived and compared with that by DG91 [13]. Disparities stem from fundamental differences between the two models in handling the velocity fields of flow. Results from the present model show that the ratio of the optimal frequency for a local maximum transport rate to the frequency of minimum phase angle of the fluid-wall interface is within the range of 1.15-1.2, which may help to guide the design of an HFV system.

For a tube with a reactive wall layer, the dispersion coefficient given in Eq. (92) for flow through a tethered tube has been shown to be independent of the wall mechanical properties (viscoelasticity, initial stresses and thickness), if the axial decay component is ignored. With some numerical calculations, the advection velocity and axial-averaged steady mass transport rate have been shown to have various degrees of dependence on the oscillation frequency, wall viscoelasticity, phase exchange parameters and initial stresses. One remarkable finding is that the magnitude of the transport rate, which represents the gas transport efficiency of $\mathrm{HFV}$, is enhanced monotonically when either the phase partitioning or the reversible exchange rate increases, although the dispersion coefficient is affected by these two factors in different manners. 


\section{Acknowledgments}

The work was supported by the Research Grants Council of the Hong Kong Special Administrative Region, China, through Project No. HKU 715609E, and also by the University of Hong Kong through the Small Project Funding Scheme under Project Code 200807176081.

\section{Appendix: The differences between present model and DG91 model}

1. A thin-walled tube model was considered by DG91 [13] for a pulmonary airway. The thin-wall model is based on some approximation assumptions, e.g., a constant wall thickness, uniform normal stress, and zero shear stress across the wall under deformation. A tube is modeled to be a thin-walled structure when its wall thickness is supposed to be infinitesimally small compared with the tube radius (the fraction being $<0.1$; see Chandran et al. [26]). However, as was pointed out by Sera et al. [23] and Montaudon et al. [24], the mean thickness of pulmonary airways is usually larger than $15 \%$ of the lumen radius, which requires one to use a thick-wall model. Based on the more general model by Ma and $\mathrm{Ng}$ [16], we here place no limits on the wall thickness, which can be a very small or a finite fraction of the tube radius.

2. The in vivo state of a pulmonary airway is inflated with a mean airway pressure and also under longitudinal tension. Although initial stresses were considered in their model, the mean pressure and longitudinal stretch are both ignored in the example calculations by DG91 [13]. As will be discussed later in this paper, even a small mean pressure can make finite effects on the gas transport during HFV.

3. DG91 [13] performed their analysis based on the Eulerian system. In fact, the time-mean fluid motion can be described more naturally and conveniently by the Lagrangian approach, as has been adopted by, e.g., Ng and Zhang [27] and Ma and $\mathrm{Ng}$ [16]. Also, as will be shown in this paper, the effective advection 
Table 1: Comparison between the present and previous works in modeling dispersion due to oscillatory flow through a tube.

\begin{tabular}{|c|c|c|c|}
\hline & DG91 [13] & $\operatorname{Ng}[17]$ & Present work \\
\hline $\begin{array}{c}\text { Method } \\
\text { of analysis }\end{array}$ & $\begin{array}{c}\text { perturbation and } \\
\text { Eulerian mean flow }\end{array}$ & $\begin{array}{l}\text { homogenization } \\
\text { method }\end{array}$ & $\begin{array}{l}\text { homogenization and } \\
\text { Lagrangian mean flow }\end{array}$ \\
\hline $\begin{array}{l}\text { Wall } \\
\text { model }\end{array}$ & $\begin{array}{l}\text { thin-walled flexible tube } \\
\text { without prestresses }\end{array}$ & rigid tube & $\begin{array}{c}\text { thick-walled flexible tube } \\
\text { with prestresses }\end{array}$ \\
\hline $\begin{array}{l}\text { Wall } \\
\text { reaction }\end{array}$ & inert tube & $\begin{array}{c}\text { reversible and } \\
\text { irreversible }\end{array}$ & $\begin{array}{c}\text { reversible and } \\
\text { irreversible }\end{array}$ \\
\hline
\end{tabular}

velocity is given by the section-averaged Lagrangian steady streaming velocity. The Lagrangian description is needed in this part of the mass transport problem.

4. DG91 [13] investigated gas transport in an inert tube without wall reactions. In fact, the lung is a place where the main activity is exchange of gases of various phases [28, 29, 30, 31]. A radial diffusion model was used for wall retention by Davidson and Schroter [32], Phillips and Kaye [33], Jayaraman et al. [34], and others. Alternatively, $\mathrm{Ng}$ [17] employed a first-order kinetic model for the phase exchange wall reaction. The relations between these two models were discussed in further detail by $\mathrm{Ng}$ and Rudraiah [18]. In this work, the models for wall absorption and retention by $\mathrm{Ng}$ [17] are adopted. Paul and Mazumder [35] recently studied transport of a reactive species in annular flow subject to these wall reactions.

5. DG91 [13] computed the time-averaged axial mass transport rate per cycle of a diffusible substance. Their mathematical expression was, however, rather lengthy and complicated, causing it not so easy to see the dependence of the mass transport mechanism on the controlling parameters. In this study, following the homogenization technique [36], we can obtain a transport equation containing simpler and explicit expressions for the effective coefficients of advection and dispersion. 


\section{References}

[1] J.B. Grotberg, Pulmonary flow and transport phenomena, Annu. Rev. Fluid Mech. 26 (1994) 529-571.

[2] J.A. Krishnan, R.G. Brower, High-frequency ventilation for acute lung injury and ARDS, Chest 118 (3) (2000) 795-807.

[3] G.I. Taylor, Dispersion of soluble matter in solvent flowing slowly through a tube, Proc. Roy. Soc. London A 219 (1953) 186-203.

[4] P.C. Chatwin, On the longitudinal dispersion of passive contaminant in oscillatory flows in tubes, J. Fluid Mech. 71 (1975) 513-527.

[5] E.J. Watson, Diffusion in oscillatory pipe flow, J. Fluid Mech. 133 (1983) 233244.

[6] J.B. Grotberg, Volume-cycled oscillatory flow in a tapered channel, J. Fluid Mech. 141 (1984) 249-264.

[7] D.A. Godleski, J.B. Grotberg, Convection-diffusion interaction for oscillatory flow in a tapered tube, J. Biomech. Eng. 110 (1988) 283-291.

[8] D.M. Eckmann, J.B. Grotberg, Oscillatory flow and mass transport in a curved tube, J. Fluid Mech. 188 (1988) 509-527.

[9] T.J. Pedley, R.D. Kamm, The effect of secondary motion on axial transport in oscillatory tube flow, J. Fluid Mech. 193 (1988) 347-367.

[10] M.K. Sharp, R.D. Kamm, A.H. Shapiro, E. Kimmel, G.E. Karniadakis, Dispersion in a curved tube during oscillatory flow, J. Fluid Mech. 223 (1991) $537-563$.

[11] Z. Zhang, C. Kleinstreuer, Transient airflow structures and particle transport in a sequentially branching lung airway model, Phys. Fluids 14 (2) (2002) 862-880.

[12] K. Adler, C. Brucker, Dynamic flow in a realistic model of the upper human lung airways, Exp. Fluids 43 (2-3) (2007) 411-423. 
[13] C.A. Dragon, J.B. Grotberg, Oscillatory flow and mass transport in a flexible tube, J. Fluid Mech. 231 (1991) 135-155.

[14] P.E. Hydon, T.J. Pedley, Axial dispersion in a channel with oscillating walls, J. Fluid Mech. 249 (1993) 535-555.

[15] P.E. Hydon, T.J. Pedley, Dispersion in oscillatory channel flow with coupled transverse wall motion, Eur. J. Mech. B/Fluids 15 (1996) 143-156.

[16] Y. Ma, C.O. Ng, Wave propagation and induced steady streaming in viscous fluid contained in a prestressed viscoelastic tube, Phys. Fluids 21 (5) (2009) 051901.

[17] C.O. Ng, Dispersion in steady and oscillatory flows through a tube with reversible and irreversible wall reactions, Proc. Roy. Soc. A 462 (2006) 481-515.

[18] C.O. Ng, N. Rudraiah, Convective diffusion in steady flow through a tube with a retentive and absorptive wall, Phys. Fluids 20 (7) (2008) 073604.

[19] D.X. Zhang, Z.M. Lu, Y.L. Liu, C.O. Ng, Numerical simulation of the dispersion in oscillating flows with reversible and irreversible wall reactions, J. Hydrodyn. 21 (4) (2009) 482-490.

[20] P.C. Fife, K.R.K. Nicholes, Dispersion in flow through small tubes, Proc. Roy. Soc. London A 344 (1975) 131-145.

[21] C.C. Mei, The Applied Dynamics of Ocean Surface Waves, World Scientific, 1989.

[22] H.B. Atabek, H.S.P. Lew, Wave propagation through a viscous incompressible fluid contained in an initially stressed elastic tube, Biophys. J. 6 (1966) 481-503.

[23] T. Sera, S. Satoh, H. Horinouchi, K. Kobayashi, K. Tanishita, Respiratory flow in a realistic tracheostenosis model, J. Biomech. Eng. 125 (4) (2003) 461-471.

[24] M. Montaudon, P. Desbarats, P. Berger, G.D. Dietrich, R. Marthan, F. Laurent, Assessment of bronchial wall thickness and lumen diameter in human adults using multi-detector computed tomography: comparison with theoretical models, J. Anat. 211 (5) (2007) 579-588. 
[25] Z.Z. Teng, I. Ochoa, Z.Y. Li, M. Doblare, Study of tracheal collapsibility, compliance and stress by considering its asymmetric geometry, Med. Eng. Phys. 31 (3) (2009) 328-336.

[26] K.B. Chandran, A.P. Yoganathan, S.E. Rittgers, Biofluid Mechanics: The Human Circulation, CRC, 2007.

[27] C.O. Ng, X. Zhang, Mass transport in water waves over a thin layer of soft viscoelastic mud, J. Fluid Mech. 573 (2007) 105-130.

[28] B.S. Mazumder, S.K. Das, Effect of boundary reaction on solute dispersion in pulsatile flow through a tube, J. Fluid Mech. 239 (1992) 523-549.

[29] Y. Jiang, J.B. Grotberg, Bolus contaminant dispersion in oscillatory tube flow with conductive walls, J. Biomech. Eng. 115 (1993) 424-431.

[30] A. Sarkar, G. Jayaraman, The effect of wall absorption on dispersion in annular flows, Acta Mech. 158 (2002) 105-119.

[31] K.K. Mondal, B.S. Mazumder, On the solute dispersion in a pipe of annular cross section with absorbing boundary, ZAMM 85 (6) (2005) 422-430.

[32] M.R. Davidson, R.C. Schroter, A theoretical model of absorption of gases by the bronchial wall, J. Fluid Mech. 129 (1983) 313-335.

[33] C.G. Phillips, S.R. Kaye, Approximate solutions for developing shear dispersion with exchange between phases, J. Fluid Mech. 374 (1998) 195-219.

[34] G. Jayaraman, T.J. Pedley, A. Goyal, Dispersion of solute in a fluid flowing through a curved tube with absorbing walls, Q. J. Mech. Appl. Math. 51 (1998) $577-598$.

[35] S. Paul, B.S. Mazumder, Transport of reactive solutes in unsteady annular flow subject to wall reactions, Eur. J. Mech. B/Fluids 28 (2009) 411-419.

[36] C.C. Mei, J.L. Auriault, C.O. Ng, Some applications of the homogenization theory, Adv. Appl. Mech. 32 (1996) 277-348. 


\section{Figure captions}

Figure 1 (a) Steady mass transport rate $\hat{m}^{\prime}$, and (b) phase lag $\phi$ of the fluid--wall interface, as computed from the present model (solid lines), and from DG91's [13] model (dashed lines), as a function of the Womersley number $\alpha$, for elasticity $\beta=$ $5,50,500,500000$, where $\gamma=0.075, \hat{b}=1.056, \mathrm{Sc}=0.882$, and $\hat{\Gamma}=\hat{\omega}=0$.

Figure 2 Values of the Womersley number $\alpha$ for the local maximum transport rate (solid line), and for the minimum interfacial phase angle (dashed line), as a function of the elasticity $\beta$, where $\hat{b}=1.056, \gamma=0.075, \mathrm{Sc}=0.882$, and $\hat{\Gamma}=\hat{\omega}=0$.

Figure 3 (a) Effective dispersion coefficient $D_{E C}$, and (b) imaginary part of the wavenumber $\hat{k}_{i}$, as a function of the Womersley number $\alpha$, for elasticity $\beta=$ $5,50,500,500000$, where $\gamma=0.075, \hat{b}=1.056$, Sc $=0.882$, and $\hat{\Gamma}=\hat{\omega}=0$.

Figure 4 Effective advection coefficient $W_{D C}$ as a function of the Womersley number $\alpha$, where $\beta=50, \gamma=0.075, \mathrm{Sc}=0.882, \hat{\omega}=\mathrm{Da}=1.0$, for (a) initial pressure $q=0-2000$, where the longitudinal stretch ratio $S=1.3$; (b) $S=1-1.4$, where $q=1000$.

Figure 5 Axial-averaged steady mass transport rate $\overline{\hat{m}^{\prime}}$ as a function of the Womersley number $\alpha$, where $\beta=50$ and $\gamma=0.075$, for (a) Sc $=0.5-2.0$, where $\hat{\omega}=\mathrm{Da}=S=1.0$, and $q=0$; (b) $\hat{\omega}=0.0-10.0$, where $\mathrm{Sc}=\mathrm{Da}=S=1.0$, and $q=0 ;(\mathrm{c}) \mathrm{Da}=0.0-10.0$, where $\mathrm{Sc}=\hat{\omega}=S=1.0$, and $q=0 ;$ (d) $q=0-2000$, where $\mathrm{Sc}=\hat{\omega}=\mathrm{Da}=1.0$, and $S=1.3$. 

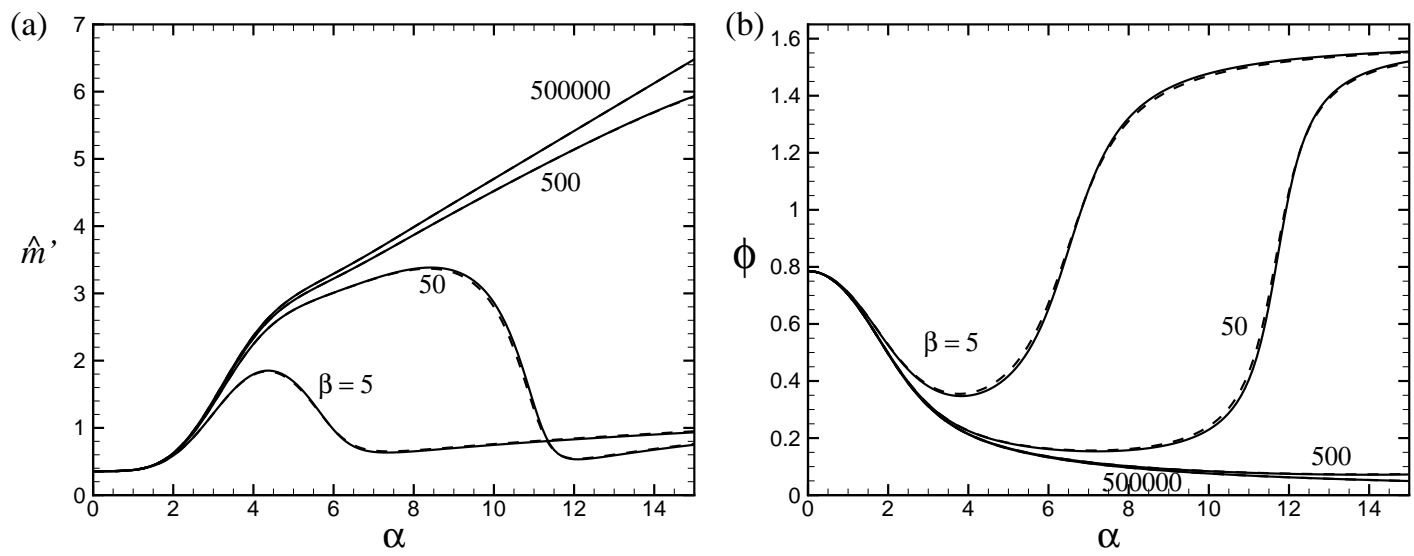

Figure 1: (a) Steady mass transport rate $\hat{m}^{\prime}$, and (b) phase lag $\phi$ of the fluid-wall interface, as computed from the present model (solid lines), and from DG91's [13] model (dashed lines), as a function of the Womersley number $\alpha$, for elasticity $\beta=$ $5,50,500,500000$, where $\gamma=0.075, \hat{b}=1.056, \mathrm{Sc}=0.882$, and $\hat{\Gamma}=\hat{\omega}=0$. 


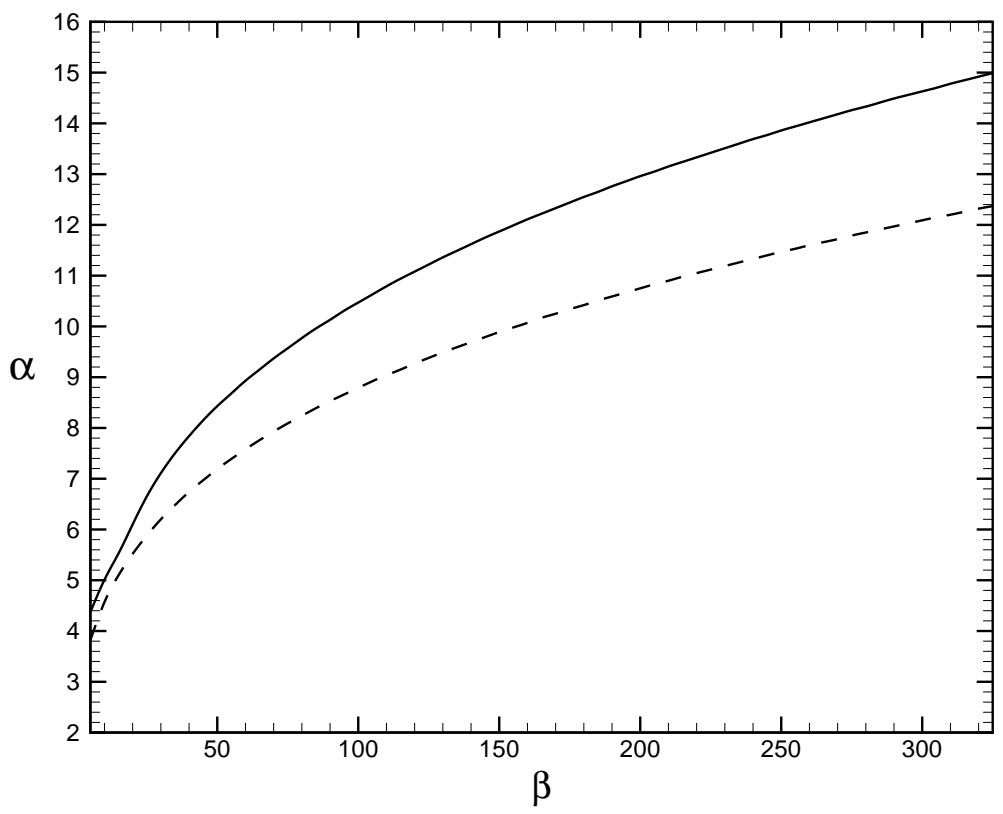

Figure 2: Values of the Womersley number $\alpha$ for the local maximum transport rate (solid line), and for the minimum interfacial phase angle (dashed line), as a function of the elasticity $\beta$, where $\hat{b}=1.056, \gamma=0.075, \mathrm{Sc}=0.882$, and $\hat{\Gamma}=\hat{\omega}=0$. 

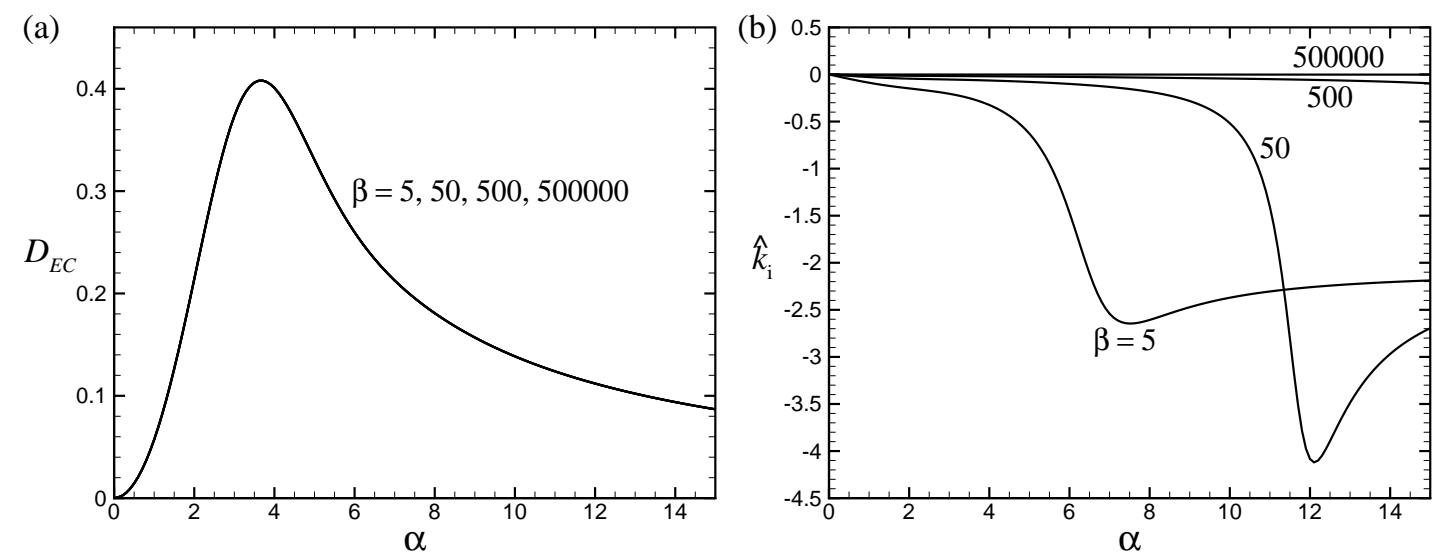

Figure 3: (a) Effective dispersion coefficient $D_{E C}$, and (b) imaginary part of the wavenumber $\hat{k}_{i}$, as a function of the Womersley number $\alpha$, for elasticity $\beta=$ $5,50,500,500000$, where $\gamma=0.075, \hat{b}=1.056$, Sc $=0.882$, and $\hat{\Gamma}=\hat{\omega}=0$. 

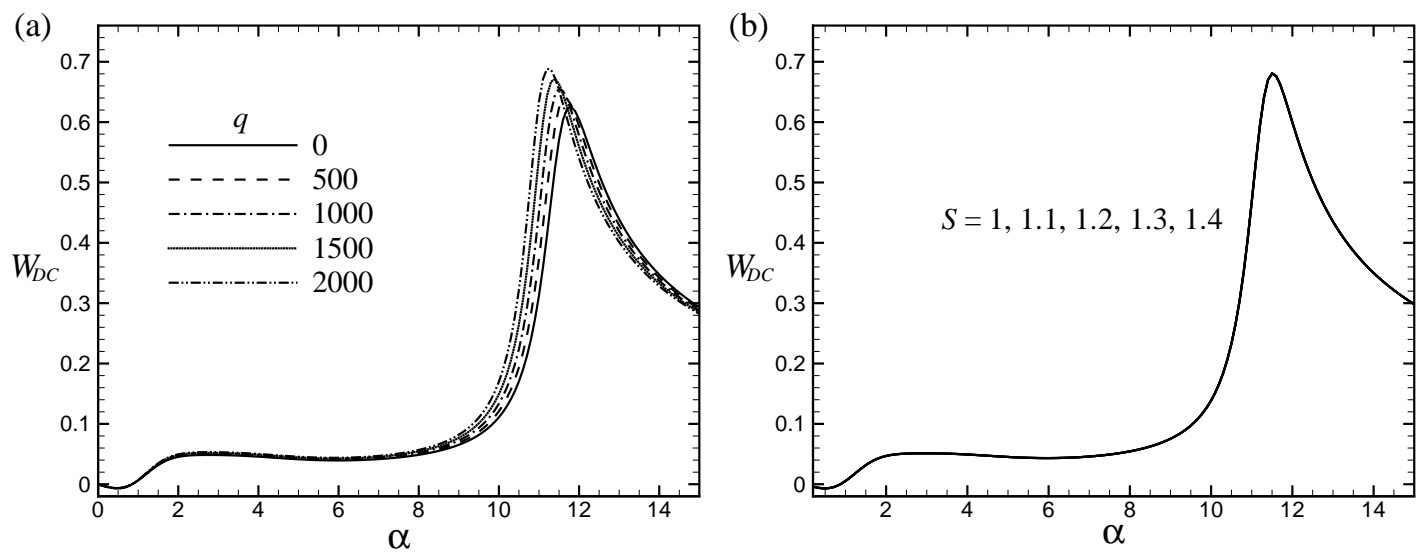

Figure 4: Effective advection coefficient $W_{D C}$ as a function of the Womersley number $\alpha$, where $\beta=50, \gamma=0.075, \mathrm{Sc}=0.882, \hat{\omega}=\mathrm{Da}=1.0$, for (a) initial pressure $q=0$ 2000, where the longitudinal stretch ratio $S=1.3$; (b) $S=1-1.4$, where $q=1000$. 

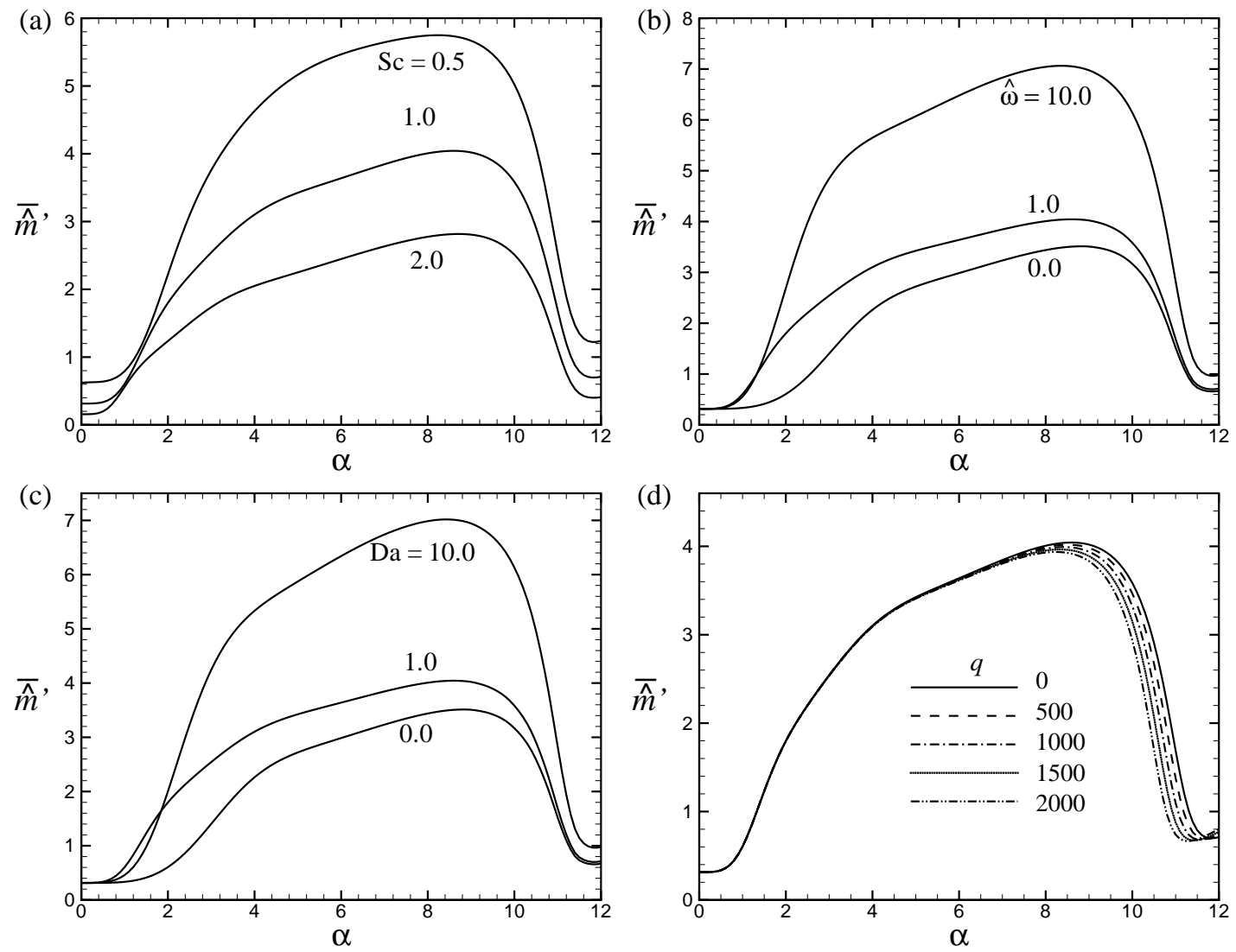

Figure 5: Axial-averaged steady mass transport rate $\overline{\hat{m}^{\prime}}$ as a function of the Womersley number $\alpha$, where $\beta=50$ and $\gamma=0.075$, for (a) Sc $=0.5-2.0$, where $\hat{\omega}=\mathrm{Da}=S=1.0$, and $q=0$; (b) $\hat{\omega}=0.0-10.0$, where $\mathrm{Sc}=\mathrm{Da}=S=1.0$, and $q=0$; (c) $\mathrm{Da}=0.0-10.0$, where $\mathrm{Sc}=\hat{\omega}=S=1.0$, and $q=0$; (d) $q=0-2000$, where $\mathrm{Sc}=\hat{\omega}=\mathrm{Da}=1.0$, and $S=1.3$. 